\title{
Macrophages Promote the Invasion of Breast Carcinoma Cells via a Colony-Stimulating Factor-1/Epidermal Growth Factor Paracrine Loop
}

\author{
Sumanta Goswami, ${ }^{1}$ Erik Sahai, ${ }^{1,4}$ Jeffrey B. Wyckoff, ${ }^{1}$ Michael Cammer, ${ }^{3}$ Dianne Cox, ${ }^{1}$ \\ Fiona J. Pixley, ${ }^{2}$ E. Richard Stanley, ${ }^{2}$ Jeffrey E. Segall, ${ }^{1}$ and John S. Condeelis ${ }^{1,3}$ \\ Departments of ${ }^{1}$ Anatomy and Structural Biology, ${ }^{2}$ Developmental and Molecular Biology, ${ }^{3}$ Analytical Imaging Facility, Albert Einstein \\ College of Medicine, Bronx, New York and ${ }^{4}$ Tumour Cell Biology Laboratory, Cancer Research UK London Research Institute, \\ London, United Kingdom
}

\begin{abstract}
Previous studies have shown that macrophages and tumor cells are comigratory in mammary tumors and that these cell types are mutually dependent for invasion. Here we show that macrophages and tumor cells are necessary and sufficient for comigration and invasion into collagen $I$ and that this process involves a paracrine loop. Macrophages express epidermal growth factor (EGF), which promotes the formation of elongated protrusions and cell invasion by carcinoma cells. Colony stimulating factor 1 (CSF-1) produced by carcinoma cells promotes the expression of EGF by macrophages. In addition, EGF promotes the expression of CSF-1 by carcinoma cells thereby generating a positive feedback loop. Disruption of this loop by blockade of either EGF receptor or CSF-1 receptor signaling is sufficient to inhibit both macrophage and tumor cell migration and invasion. (Cancer Res 2005; 65(12): 5278-83)
\end{abstract}

\section{Introduction}

Tumor cell motility and invasion is required for tumors to metastasize from their site of origin. Chemotactic factors found in the tumor microenvironment can promote the motility and invasion of tumor cells and therefore facilitate metastasis. Epidermal growth factor (EGF) is a potent chemotactic factor for many cells of epithelial origin $(1,2)$ and is expressed in many tumor types (3). Increased expression of EGF receptors correlates with poor prognosis in human cancers and is sufficient to promote metastasis in animal models (4). Several lines of evidence suggest that EGF is produced by nontumor cell types; infiltrating T lymphocytes found in ovarian and breast tumors can produce heparin binding EGF (5); and analysis of breast tumor explants revealed that EGF is produced by tumor-associated macrophages (TAM) and not by carcinoma cells $(6,7)$. TAMs correlate with poor prognosis in human breast cancer $(8,9)$, and mouse mammary tumor metastasis is attenuated in animals with reduced numbers of macrophages (10). In addition, high levels of colony-stimulating factor 1 (CSF-1), which promotes macrophage differentiation and survival, correlate with poor prognosis in certain tumor types (11).

Note: Supplementary data for this article are available at Cancer Research Online (http://cancerres.aacrjournals.org/)

S. Goswami and E. Sahai contributed equally to this work.

Requests for reprints: Sumanta Goswami, Department of Anatomy and Structural Biology, Albert Einstein College of Medicine, 1300 Morris Park Avenue, Bronx, NY 10461. Phone: 718-430-4453; Fax: 718-430-8996; E-mail: sgoswami@aecom.yu.edu.

(c)2005 American Association for Cancer Research.
By using an "in vivo invasion assay" to collect invasive cells from experimental mammary tumors, we have recently shown that both carcinoma cells and macrophages comigrate and depend upon each other to be invasive in vivo (12). The invasion of carcinoma cells can be blocked by inhibiting either the EGF receptor (EGFR) or the CSF-1 receptor (CSF-1R) which are expressed by carcinoma cells and macrophages, respectively. This in vivo study suggested that a paracrine loop exists between carcinoma cells and macrophages but an in vitro analysis of the interactions of these cell types during invasion enables a more controlled evaluation of paracrine loop. In this study, we show that macrophages promote the directed motility and invasion of carcinoma cells in the absence of other cell types and exogenous growth factors. Furthermore, we show that this increase in invasion is dependent on EGF produced by macrophages and the CSF-1 produced by the carcinoma cells, EGFR function in the carcinoma cells, and CSF-1R function in the macrophages. EGF expression by macrophages is promoted by CSF-1, and CSF-1 expression by carcinoma cells is promoted by EGF, leading to a positive feedback in the paracrine loop.

\section{Materials and Methods}

Cell culture. MTLn3-GFP and RAW264.7 (LR5) cells were cultured in $\alpha$-MEM with 5\% fetal bovine serum (FBS). BAC1.2F51.2F5 cells were cultured in $\alpha$-MEM with $10 \%$ FBS and $36 \mathrm{ng} / \mathrm{mL}$ of Human recombinant CSF-1 (a gift from Chiron Corp., Emeryville, CA).

Carcinoma/macrophage coculture. MTLn3-GFP $(n=50,000)$ were plated on a $35-\mathrm{mm}$ MatTek Dish in the presence or absence of 250,000 BAC1.2F51.2F5 cells in $2 \mathrm{~mL} \alpha$-MEM with $10 \%$ FBS and $36 \mathrm{ng} / \mathrm{mL}$ CSF-1. After 16 hours, epifluorescence images were taken of 20 fields chosen at random. The number of MTLn3-GFP cells whose length was more than four times their width was counted and compared against the total number of cells in the field. Where indicated, cells were treated with $1 \mu \mathrm{mol} / \mathrm{L}$ Iressa (an EGFR tyrosine kinase inhibitor) dissolved in DMSO, or $25 \mu \mathrm{g} / \mathrm{mL}$ CSF1R blocking antibody (AFS98, ref. 13, a gift from Dr. S. Nishikawa, Kyoto University, Japan), or $25 \mu \mathrm{g} / \mathrm{mL}$ EGF blocking antibody (Upstate \#06-102). Vehicle controls containing DMSO or F4/80 antibody were treated in a similar manner. Time lapse microscopy was done at $37^{\circ} \mathrm{C}$ and cultures were overlaid with mineral oil to prevent changes in $\mathrm{pH}$.

Collagen invasion assay. MTLn3-GFP or MDA-MB231 cells $(n=80,000)$ were plated on a 35-mm MatTek Dish $\left(\sim 80\right.$ cells $\left./ \mathrm{mm}^{2}\right)$ in the presence or absence of 200,000 BAC1.2F51.2F5 cells in $2 \mathrm{~mL} \alpha$-MEM with $10 \%$ FBS and $36 \mathrm{ng} / \mathrm{mL}$ of CSF-1. After 16 hours, cells were overlaid with a $750-$ to $1,000-\mu \mathrm{m}$ layer of 5 to $6 \mathrm{mg} / \mathrm{mL}$ collagen I, which was allowed to gel for 90 minutes before the addition of $1 \mathrm{~mL} \alpha$-MEM with $10 \%$ chemically defined lipid mix (GIBCO 11905-031) and insulin-transferrin-selenium (GIBCO 41400-045). Where indicated cells were treated with $1 \mu \mathrm{mol} / \mathrm{L}$ Iressa (an EGFR tyrosine kinase inhibitor) dissolved in DMSO, or CSF-1R blocking antibody (AFS98) at $10 \mu \mathrm{g} / \mathrm{mL}$, or EGF blocking antibody (Upstate \#06-102). Vehicle controls containing DMSO or F4/80 antibody were treated in 
a similar manner. Cells were pretreated with antibodies for 2 hours before being overlaid with collagen. After 24 hours, the assay was fixed with $4 \%$ formaldehyde and analyzed by confocal microscopy: optical z-sections were taken every $5 \mu \mathrm{m}$ starting at the base of the dish and extending at least $50 \mu \mathrm{m}$ into the collagen gel. To quantify the invasion of MTLn3-GFP cells the green fluorescence protein (GFP) fluorescence in the z-sections from $20 \mu \mathrm{m}$ into the collagen and above was added up and divided by the sum of GFP fluorescence in all the z-sections. To quantify the invasion of the MDAMB231 cells the number of cells above $20 \mu \mathrm{m}$ (1.5 cell diameters) into the collagen was counted and divided by the total number of cells in all the z-sections. The data shown represents analysis of $\geq 200$ cells with data collected from at least three independent experiments.

RNA extraction and PCR amplification. RNA Extraction was done using the RNeasy kit (Qiagen, Valencia, CA) in accordance with the manufacturer's protocol; RNA was eluted with $30 \mu \mathrm{L}$ RNase-free water. The total RNA was reverse-transcribed directly using the superscript cDNA synthesis kit (Clontech, Palo Alto, CA) according to the manufacturer's protocol. PCR was done using specific primers for each of the growth factors and their receptors (see Supplementary Table for primer sequences, amplicon size, and annealing temperature). Electrophoresis of the PCR products was done on $2 \%$ agarose gel and visualized by ethidium bromide staining.

Real-time PCR confirmation. Quantitative reverse transcription-PCR (QRT-PCR) analysis was done to determine the change of expression of EGF in BAC1.2F51.2F5 cells cultured with or without $36 \mathrm{ng} / \mathrm{mL} \mathrm{CSF-1} \mathrm{for}$ 16 hours using the ABI 7900 (Applied Biosystems, Foster City, CA) with sequence-specific primer pairs for EGF (see Supplementary Table for primer sequences, amplicon size, and annealing temperature) as described previously (14). Similarly, QRT-PCR analysis was done to determine the change of expression of CSF-1 in MTLn3 cells cultured with or without $1 \mathrm{nmol} / \mathrm{L}$ EGF for 4 hours. The SYBR Green PCR Core Reagents system (Perkin-Elmer Applied Biosystems) was used for real-time monitoring of amplification. Results were evaluated with the ABI Prism SDS 2.0 software. Both the genes tested for regulation were compared with two housekeeping genes $(\beta$-actin and $G A P D H)$ for the analysis.

\section{Results}

To determine if macrophages can affect the behavior of carcinoma cells, we did coculture experiments using MTLn3 mammary carcinoma cells and either BAC1.2F51.2F5 (BAC1.2F5; ref. 15) or RAW264.7 (LR5) macrophages (16). Figure $1 A$ shows that when cultured alone, MTLn3 have a nonpolarized, well-spread morphology. In contrast, when cultured with either BAC1.2F51.2F5 or LR5 macrophages, $\sim 25 \%$ of the cells had elongated protrusions. The formation of these protrusions required the macrophage growth factor CSF-1; if CSF-1 was not present then macrophages only moderately stimulated the formation of elongated protrusions. Figure $1 B$ shows that CSF-1 had no effect on the morphology of MTLn3 cells in the absence of macrophages suggesting that CSF-1 is not signaling directly to the carcinoma cells but indirectly via the macrophages.

We did time lapse microscopy to determine if the changes in carcinoma cell morphology induced by macrophages affect cell motility. The movement of MTLn3 cells either cultured alone or with BAC1.2F5 macrophages in the presence of CSF-1 was recorded for 1 hour. MTLn3 cells cultured alone are highly motile but move in a random manner with frequent changes in direction (Fig. $1 C$ and $D$; Supplementary Movie 1). In contrast, the elongated protrusions observed in MTLn3 cells cultured with macrophages are associated with an increase in the net amount of cell movement (Fig. 1C; Supplementary Movie 2). To quantify the directedness of cell motility, we did morphometric analysis (DIAS). The directionality of movement was calculated by dividing the net distance moved by the centroid of the cell in 1 hour by the total distance moved in the same period. A cell moving in a linear path will have a value of 1 , whereas a cell moving randomly will have a value close to 0 . Figure $1 D$ shows that carcinoma cells move with significantly more directionality when cultured with macrophages. The average speed of cell movement decreased significantly from 0.61 to $0.40 \mu \mathrm{m} / \mathrm{min}$ in the presence of macrophages. Furthermore, the speed/net path decreased from 0.034 to 0.017 when MTLn3 cells were cultured with macrophages.

This latter measurement shows a decrease in the frequency of direction changes, indicating that the carcinoma cells move in
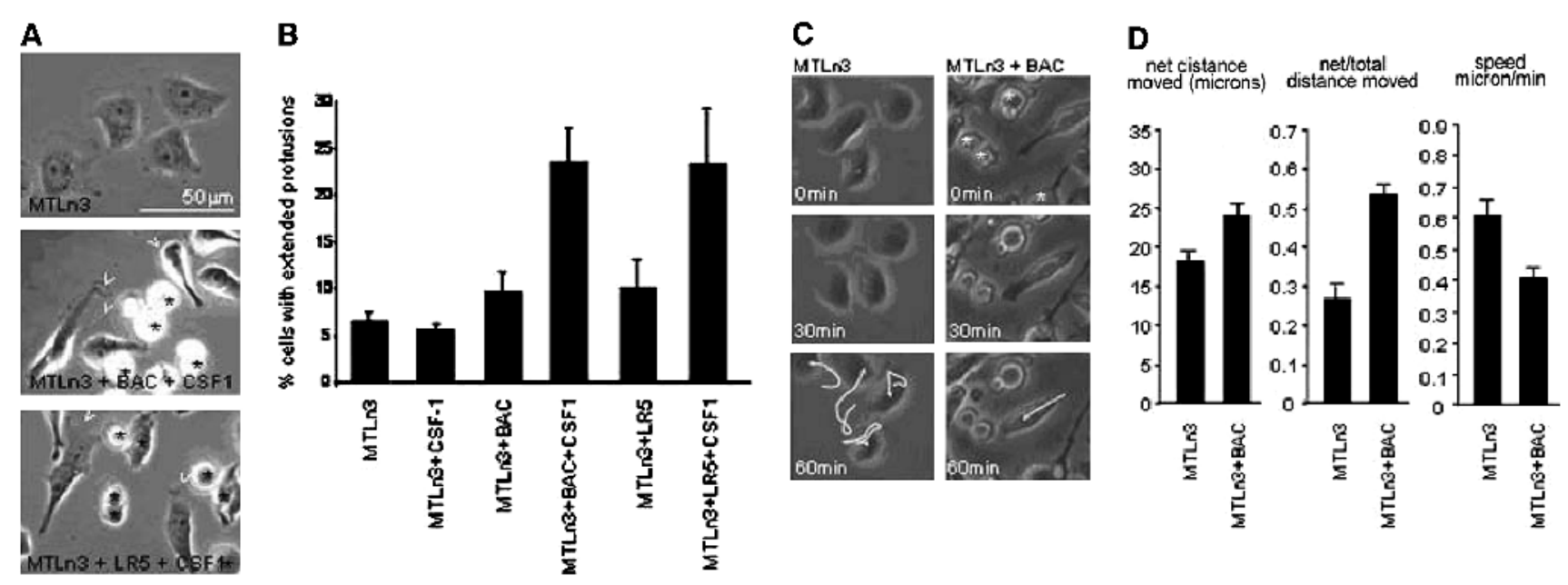

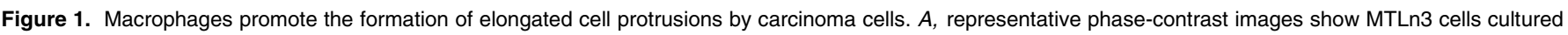

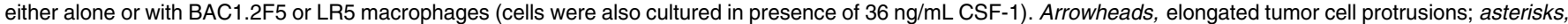
macrophages in phase-contrast image. $B$, quantification of the proportion of GFP-MTLn3 carcinoma cells with elongated protrusions when cultured alone, with BAC1.2F5 macrophages, or with LR5 macrophages. Columns, averages of two or more experiments; bars, SE. $C$, representative images of a time lapse video of MTLn3 cocultured with BAC1.2F5 macrophages and $36 \mathrm{ng} / \mathrm{mL}$ CSF1. Asterisk, BAC1.2F5 cell. $D$, time lapse videos of MTLn3 cells cultured either alone or with

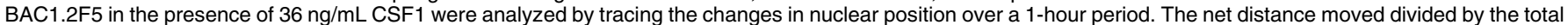
distance moved is plotted for cells from three experiments. Significantly different $P<0.005$ (two-tailed unequal variance Student's $t$ test). 
a directed fashion towards the macrophages. An explanation for the reduction in speed is that the carcinoma cells become polarized in the proximity of macrophages, their random movements are minimized, and eventually they stop moving.

We next set out to investigate if the more directed movement of carcinoma cells in the presence of macrophages was associated with increased invasive behavior. The margins of breast tumors are surrounded by collagen-rich tissues. In addition, tumor cells can migrate on collagen fibers to blood vessels where they intravasate (17). To mimic this environment, we overlaid cocultures of carcinoma cells and macrophages with a collagen I gel. (Fig. $2 A$ shows a schematic representation of the experimental setup). After 24 hours, the proportion of cells invading at least $20 \mu \mathrm{m}$ into the collagen was determined by taking optical sections with a confocal microscope. When cultured alone, $<5 \%$ of the MTLn3 cells invade in to the collagen I gel; whereas in the presence of BAC1.2F51.2F5 macrophages, $\sim 25 \%$ of the MTLn3 cells invade $20 \mu \mathrm{m}$ into the collagen (Fig. $2 B$ and $C$ ). Interestingly, exogenous CSF-1 was not required for the increase in invasion; this may be because the collagen gel limits the diffusion of CSF-1 produced by the carcinoma cells (see below). Invasive carcinoma cells and macrophages were often found in within $50 \mu \mathrm{m}$ of each other in the collagen gel suggesting that close proximity of the two cell types is required for their invasive behavior (Fig. 2B). LR5 macrophages also promoted the invasion of MTLn3, albeit to a lesser extent than BAC1.2F51.2F5 macrophages (Fig. 2C). We also tested if MDA-MB 231 human breast carcinoma cells were more invasive in the presence of macrophages. Figure $2 D$ shows that MDA-MB 231 cells are moderately invasive when cultured alone but become highly invasive when cultured in the presence of BAC1.2F5 cells. Coculture of MTLn3 cells with dermal fibroblasts did not increase the invasion of MTLn3 cells (data not shown). These data clearly show that macrophages are able to promote the invasion of carcinoma cells into a three-dimensional collagen matrix.

Our in vivo analyses have shown that EGFR signaling is crucial for mammary carcinoma cell motility (12). To test the role of EGFR signaling directly, we treated cells with Iressa that specifically inhibits the tyrosine kinase activity of EGFR (18). Figure 3 shows that treatment of carcinoma-macrophage cocultures with Iressa prevents the formation of elongated protrusions and invasion into collagen by MTLn3 cells (Fig. $3 A$ and $B$ ). Similar results were found using PD153035 and tyrophostin AG1478 to block EGFR function (data not shown). Iressa treatment also reduced the macrophagestimulated invasion of MDA-MB 231 cells (Fig. 3C). RT-PCR analysis showed that EGFR (ErbB1) was expressed by carcinoma cells but not by macrophages (Fig. $4 A$ ). These data show that EGFR function is required in carcinoma cells for macrophages to stimulate to their invasion.

The increased invasion of MTLn3 cells when cocultured with macrophages occurs in the absence of exogenously applied EGF family ligands. We therefore sought to investigate which EGFR ligand might be responsible for activating EGFR and promoting invasion. RT-PCR analysis showed that both the macrophages and MTLn3 cells expressed transforming growth factor $\alpha$ (TGF- $\alpha$ ), heparin-binding EGF (HB-EGF), and amphiregulin. On the other hand, EGF was only expressed by the macrophages and CSF-1 only by the MTLn3 cells (Fig. $4 A$ ). The results outlined in Fig. 1 show that the ability of macrophages to affect the morphology of carcinoma cells is greatly increased by CSF-1. This suggests that CSF-1 promotes the expression of factors that influence the morphology of carcinoma cells. We therefore investigated if CSF-1 increased the expression of EGFR ligands by BAC1.2F5 macrophages. EGF expression in macrophages was increased by CSF-1 treatment, whereas the expression of other EGF family members

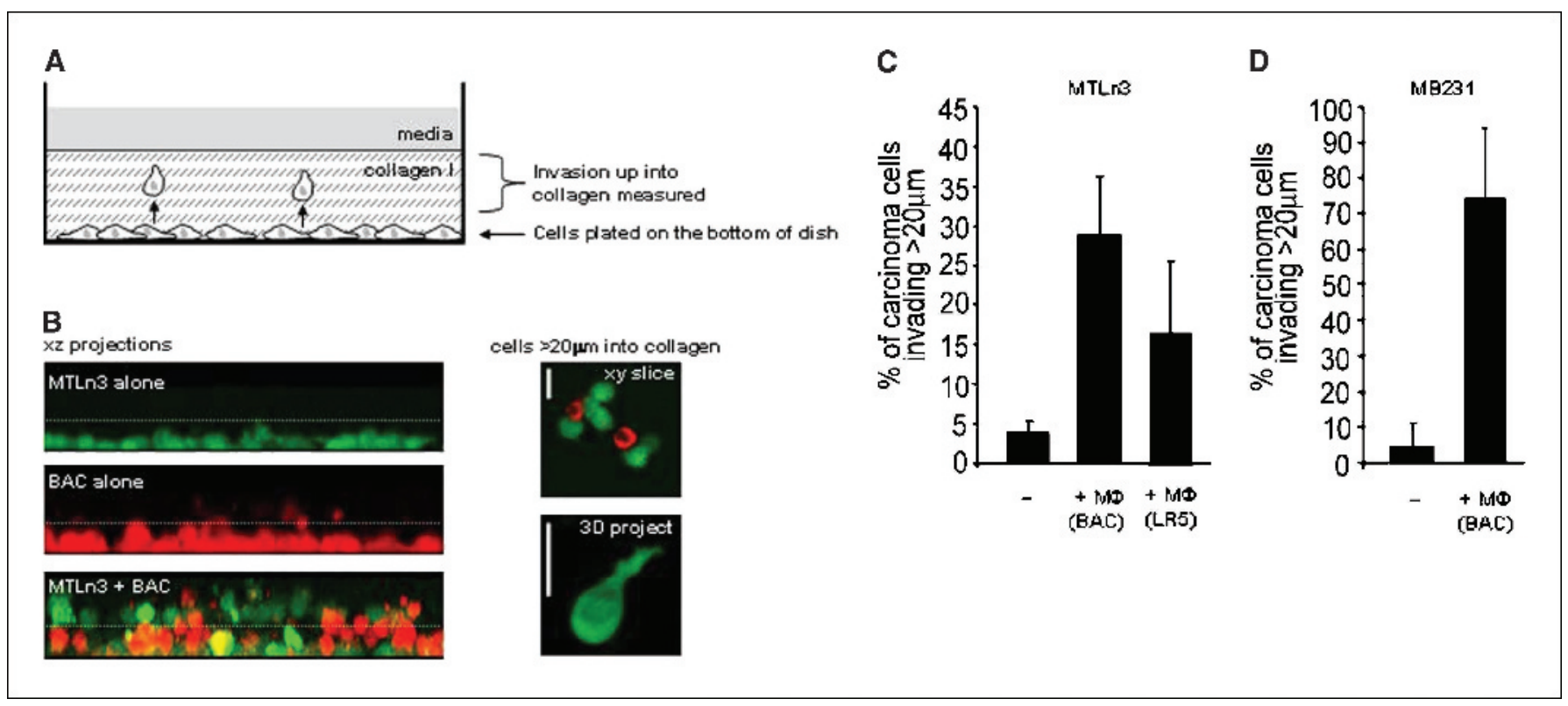

Figure 2. Macrophages promote the invasion of carcinoma cells into a collagen matrix. $A$, diagram of the experimental setup. Cells are plated on the bottom of a

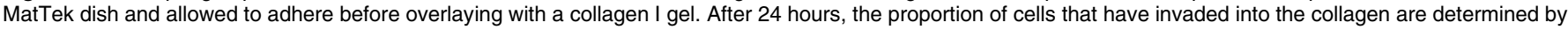

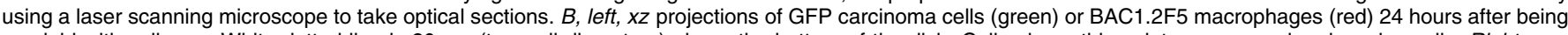
overlaid with collagen. White dotted line is $20 \mu \mathrm{m}$ (two cell diameters) above the bottom of the dish. Cells above this point were scored as invasive cells. Right, representative $x y$ image of cells $60 \mu \mathrm{m}$ above the bottom of the dish. Note the proximity of migrating macrophages and carcinoma cells. $C$, quantification of the proportion of invasive (above $20 \mu \mathrm{m}$ ) MTLn3 carcinoma cells either plated alone or with BAC1.2F5 or LR5 macrophages. Columns, averages of three or more experiments; bars, SE. D, quantification of the proportion of invasive (above $20 \mu \mathrm{m}$ ) BAC1.2F5 macrophages either plated alone or with MTLn3 carcinoma cells. Columns, averages of three or more experiments; bars, SE. 


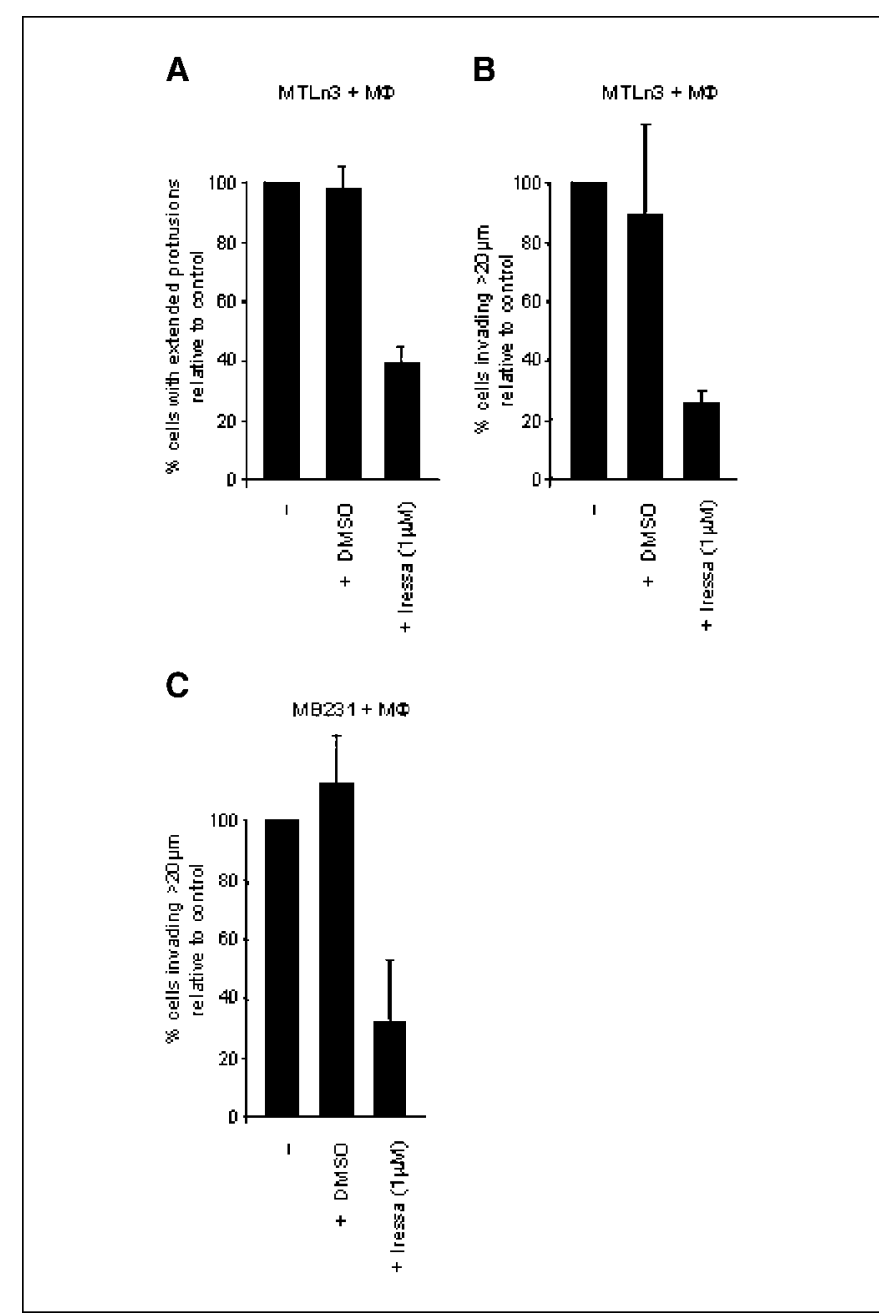

Figure 3. Macrophage-induced cell protrusions and invasion requires EGFR function. $A$, quantification of the percentage of GFP-MTLn3 carcinoma cells with elongated protrusions when cultured with BAC1.2F5 macrophages and $36 \mathrm{ng} / \mathrm{mL}$ CSF1 and treated with $1 \mu \mathrm{mol} / \mathrm{L}$ Iressa. Columns, averages of two or more experiments, \% age is relative to control MTLn3 cocultured with BAC1.2F5 cells; bars, SE. B, quantification of the \% age of invasive (above $20 \mu \mathrm{m}$ ) MTLn3 carcinoma cells when plated with BAC1.2F5 macrophages and treated with $1 \mu \mathrm{mol} / \mathrm{L}$ Iressa. Columns, averages of three experiments, $\%$ age is relative to control MTLn3 cocultured with BAC1.2F5 cells; bars, SE. $C$, quantification of the $\%$ age of invasive (above $20 \mu \mathrm{m}$ ) MDA MB231 carcinoma cells when plated with BAC1.2F5 macrophages and treated with $1 \mu \mathrm{mol} / \mathrm{L}$ Iressa. Columns, averages of three experiments, $\%$ age is relative to control MTLn3 cocultured with BAC1.2F5 cells; bars, SE.

was not dramatically altered (Fig. $4 B$ ). These data suggest that increased EGF production in the presence of CSF-1 may mediate the EGFR dependent increase in carcinoma cells with elongated protrusions observed when carcinoma cells are cocultured with macrophages. Exogenous CSF-1 is not required for macrophages to promote cell invasion suggesting either that this process is CSF-1-independent or that CSF-1 is produced by one or both cell types. In support of the latter hypothesis, we found that carcinoma cells express CSF-1 (Fig. $4 A$ ); furthermore, treatment of carcinoma cells with EGF increased CSF-1 expression (Fig. 4C). In agreement with previous work, we found that macrophages express the CSF-1R.

The results described above suggest that positive feedback is likely to promote the expression of CSF-1 and EGF when macrophages and carcinoma cells are cocultured. To test if EGF produced by macrophages is responsible for the altered morphology and increased invasion of carcinoma cells, we used blocking antibodies against the different EGFR ligands. Figure 5 shows that treatment of macrophage-carcinoma cocultures with a function blocking EGF antibody reduced the number of cells with extended protrusions and the extent of invasion (Fig. 5). Blocking antibodies to other EGF family members, amphiregulin, TGF- $\alpha$, and HB-EGF, did not affect the morphology or invasion of carcinoma cells cultured in the presence of macrophages (Supplementary Fig. 1).

The data described in Fig. 4 suggests that EGF expression by macrophages is dependent upon CSF-1 signaling: if this is correct then blockade of CSF-1R function should reduce EGF expression by macrophages and reduce the number of carcinoma cells with elongated protrusions and cell invasion. Treatment of carcinomamacrophage cocultures with function blocking anti-CSF-1R antibody AFS98, but not a control antibody reduced both the formation of elongated protrusions by MTLn3 cells and their invasion into collagen (Fig. $5 A$ and $B$ ). These data show that the ability of macrophages to stimulate the invasive behavior of carcinoma cells depends on CSF-1R signaling in macrophages and EGF signaling through EGFR in carcinoma cells.

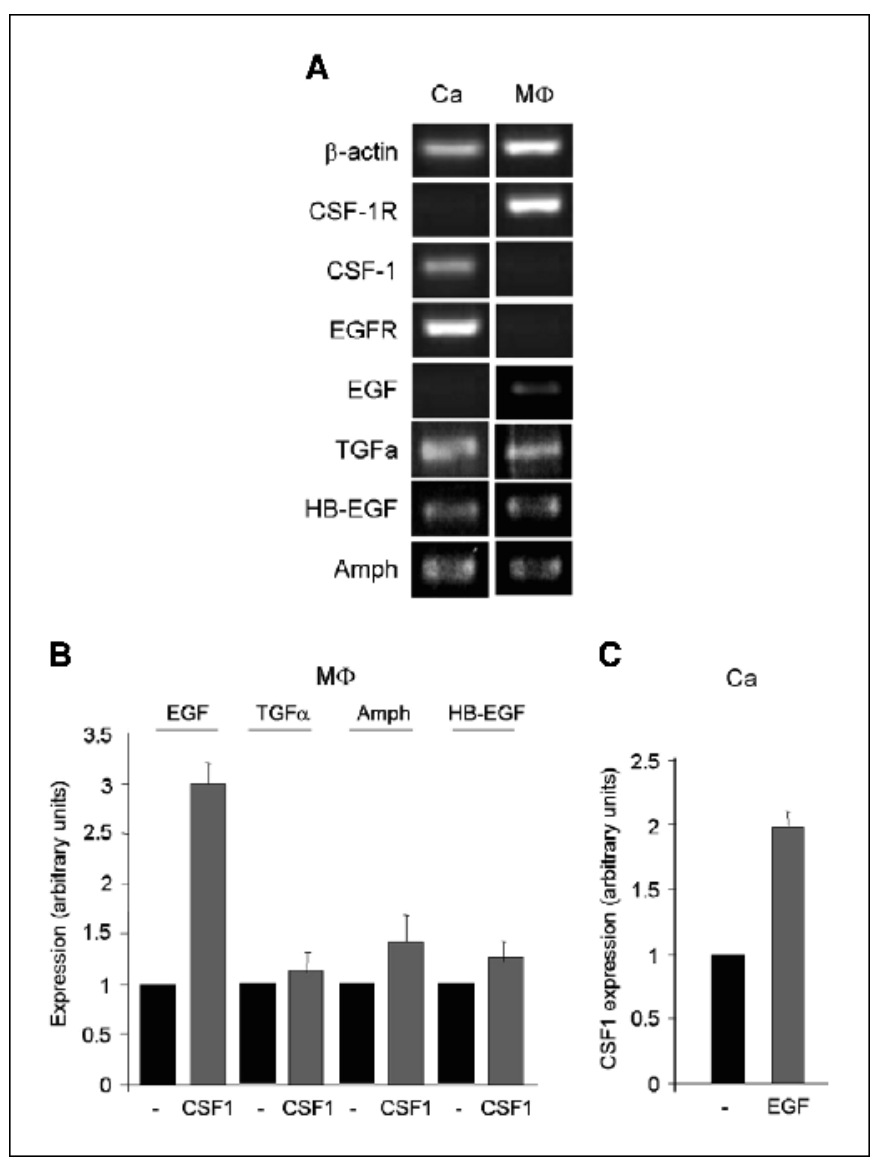

Figure 4. Expression of chemotactic factors and their receptors by carcinoma cells and macrophages. $A$, RNA was isolated from carcinoma cells and macrophages and analyzed by RT-PCR for the presence of $\beta$-actin, EGF, Amphiregulin (Amph), HB-EGF, TGF- $\alpha$, EGFR, CSF-1, and CSF-1R mRNA. $B$, RNA was isolated from macrophages cultured in the presence or absence of $36 \mathrm{ng} / \mathrm{mL}$ CSF-1 and analyzed by quantitative real time RT-PCR for the levels of EGF, TGF- $\alpha$, HB-EGF, and amphiregulin. $C$, RNA was isolated from carcinoma cells cultured in the presence or absence of $12.5 \mathrm{nmol} / \mathrm{L}$ EGF and analyzed by quantitative real-time RT-PCR for the levels of CSF-1. 


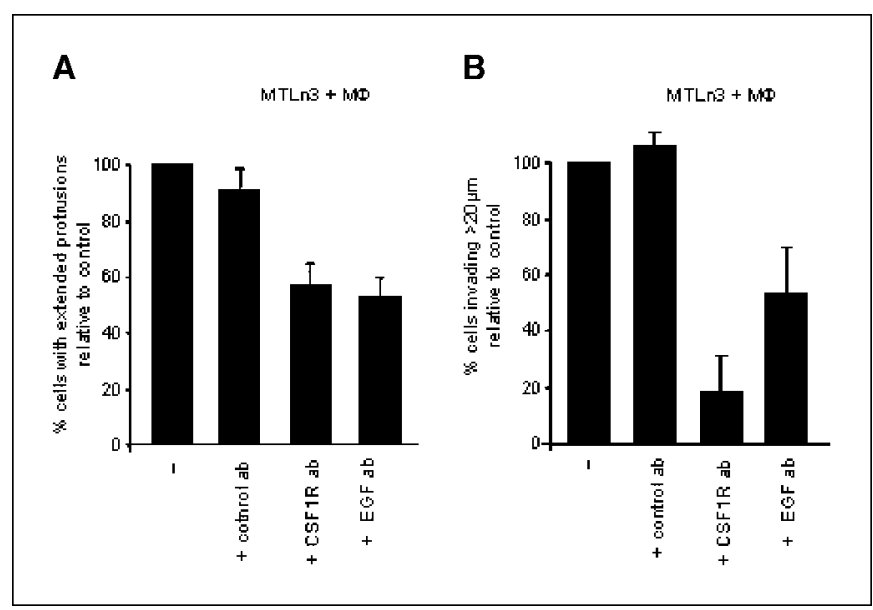

Figure 5. Macrophage-induced cell protrusions and invasion requires EGF and CSF-1R. $A$, quantification of the percentage of GFP-MTLn3 carcinoma cells with elongated protrusions when cultured with $\mathrm{BAC} 1.2 \mathrm{~F} 5$ macrophages and $36 \mathrm{ng} / \mathrm{mL}$ CSF1 and treated with either EGF or CSF-1R blocking antibodies or a control antibody. Columns, averages of two or more experiments, \% age is relative to control MTLn3 cocultured with BAC1.2F5 cells; bars, SE. B, quantification of the $\%$ age of invasive MTLn3 carcinoma cells (above $20 \mu \mathrm{m}$ ) when plated with BAC1.2F5 macrophages and treated with either EGF or CSF-1R blocking antibodies or a control antibody. Columns, averages of two or more experiments, $\%$ age is relative to control MTLn3 cocultured with BAC1.2F5 cells; bars, SE.

\section{Discussion}

Tumor cell motility is required for the metastatic process. Here we show that the paracrine loop between carcinoma cells and macrophages is necessary and sufficient for tumor cell invasion. The ability of macrophages to promote the formation of elongated protrusions and invasion of carcinoma cells is dependent on EGFR function in the carcinoma cells. These results are consistent with the wealth of data implicating members of the EGFR family in the progression of carcinomas (3). We find that EGF is expressed by macrophages but not carcinoma cells and that EGF is required to promote carcinoma cell invasion in coculture with macrophages in vitro. Other EGF family ligands were not necessary for invasion or for macrophages to affect the behavior of the carcinoma cells. Furthermore, we show that CSF-1 signaling is required for macrophages to affect the morphology and invasion of carcinoma cells. CSF-1 is expressed by the carcinoma cells and specifically promotes the expression of EGF by macrophages. Thus, the carcinoma cells promote the production of EGF by macrophages, which then promotes the invasion of the carcinoma cells. Positive feedback is generated in this paracrine loop because macrophages express more EGF when cultured in the presence of CSF-1 and carcinoma cells express more CSF-1 when EGF signaling is activated. This self-reenforcing loop may serve to recruit mono- cytes into the tumor. Consistent with this idea, EGFR-positive breast tumors recruit higher numbers of macrophages (19). Other studies have suggested that matrix metalloproteases (MMP) produced by macrophages may increase the invasion of tumor cells $(20,21)$. However, inhibition of MMPs in our assay did not significantly reduce the amount of invasion. These studies do not rule out the secretion of additional proinvasive factors by macrophages.

Addition of exogenous EGF or media conditioned by macrophages to MTLn3 cells did not promote the formation of elongated protrusions or the invasion of carcinoma cells into collagen (data not shown). We speculate that the formation of elongated protrusions and cell invasion requires asymmetrical activation of EGF signaling in cells, which is triggered in cells exposed to gradients of EGF. Macrophages secreting EGF will generate gradients and we noted that elongated protrusions are often oriented towards macrophages. Furthermore, cells moving in response to a gradient will be moving in a more directed manner and this is exactly what we observed in carcinoma cells cocultured with macrophages.

These findings support our in vivo observations that carcinoma cells and macrophages constitute the invasion population of cells found in mammary tumors (12) and provide an explanation for the correlation of high levels of CSF-1 and TAMs with poor prognosis in breast cancer $(7,8)$. TAMs are often found clustered in hotspots in particularly invasive regions of tumors and surrounding ducts $(9,22)$. We propose that macrophages promote invasive tumor cell behavior at these sites and this ultimately leads to increased metastasis. The ability of macrophages to promote the invasive behavior of other cell types is not restricted to tumors; macrophages can also promote fibroblast invasion into cartilage in rheumatoid arthritis (23).

This study shows that a paracrine loop between carcinoma cells and macrophages leads to increased carcinoma cell invasion (Fig. 5). Strategies that disable this loop may be of clinical benefit in the treatment of cancer. One attractive possibility is targeting aspects of macrophage behavior to reduce tumor cell invasion, because macrophages are unlikely to develop multidrug resistance and are genetically stable. Indeed, we have shown that interference with CSF-1R function reduces MTLn3 carcinoma cell invasion despite the fact that CSF-1R is not expressed by MTLn3 cells. Furthermore, recent work has shown that targeting CSF-1 production has antitumor effects in vivo (24).

\section{Acknowledgments}

Received 5/26/2004; revised 1/13/2005; accepted 2/18/2005.

The costs of publication of this article were defrayed in part by the payment of page charges. This article must therefore be hereby marked advertisement in accordance with 18 U.S.C. Section 1734 solely to indicate this fact.

\section{References}

1. Segall JE, Tyerech S, Boselli L, et al. EGF stimulates lamellipod extension in metastatic mammary adenocarcinoma cells by an actin-dependent mechanism. Clin Exp Metastasis 1996;14:61-72.

2. Wilson SE, He YG, Weng J, Zieske JD, Jester JV, Schultz GS. Effect of epidermal growth factor, hepatocyte growth factor, and keratinocyte growth factor, on proliferation, motility and differentiation of human corneal epithelial cells. Exp Eye Res 1994;59:665-78.

3. Mendelsohn J, Baselga J. The EGF receptor family as targets for cancer therapy. Oncogene 2000;19: 6550-65.

4. Wells A. EGF receptor. Int J Biochem Cell Biol 1999;31: 637-43.

5. Peoples GE, Blotnick S, Takahashi K, Freeman MR, Klagsbrun M, Eberlein TJ. T lymphocytes that infiltrate tumors and atherosclerotic plaques produce heparin-binding epidermal growth factor-like growth factor and basic fibroblast growth factor: a potential pathologic role. Proc Natl Acad Sci U S A 1995;92: 6547-51.

6. O’Sullivan C, Lewis CE, Harris AL, McGee JO. Secretion of epidermal growth factor by macrophages associated with breast carcinoma. Lancet 1993;342:148-9.

7. Pollard JW. Tumour-educated macrophages promote tumour progression and metastasis. Nat Rev Cancer 2004; $4: 71-8$

8. Leek RD, Lewis CE, Whitehouse R, Greenall M, Clarke J, Harris AL. Association of macrophage infiltration with angiogenesis and prognosis in invasive breast carcinoma. Cancer Res 1996;56:4625-9.

9. Goede V, Brogelli L, Ziche M, Augustin HG. Induction of inflammatory angiogenesis by monocyte chemoattractant protein-1. Int J Cancer 1999;82:765-70. 
10. Lin EY, Nguyen AV, Russell RG, Pollard JW. Colony-stimulating factor 1 promotes progression of mammary tumors to malignancy. J Exp Med 2001; 193:727-40.

11. Sapi E, Kacinski BM. The role of CSF-1 in normal and neoplastic breast physiology. Proc Soc Exp Biol Med 1999;220:1-8.

12. Wyckoff JB, Wang W, Lin EY, et al. A paracrine loop between tumor cells and macrophages is required for tumor cell migration in mammary tumors. Cancer Res 2004:64:7022-9.

13. Sudo $T$, Nishikawa $S$, Ogawa $M$, et al. Functional hierarchy of c-kit and c-fms in intramarrow production of CFU-M. Oncogene 1995;11:2469-76.

14. Wang W, Wyckoff JB, Frohlich VC, et al. Single cell behavior in metastatic primary mammary tumors correlated with gene expression patterns revealed by molecular profiling. Cancer Res 2002;62:6278-88.

15. Morgan C, Pollard JW, Stanley ER. Isolation and characterization of a cloned growth factor dependent macrophage cell line, BAC1.2F5. J Cell Physiol 1987;130: $420-7$.

16. Cox D, Chang P, Zhang O, Reddy PG, Bokoch GM Greenberg S. Requirements for both Racl and Cdc42 in membrane ruffling and phagocytosis in leukocytes. J Exp Med 1997;186:1487-94.

17. Condeelis J, Segall JE. Intravital imaging of cel movement in tumours. Nat Rev Cancer 2003;3:921-30.

18. Anderson NG, Ahmad T, Chan K, Dobson R, Bundred NJ. ZD1839 (Iressa), a novel epidermal growth factor receptor (EGFR) tyrosine kinase inhibitor, potently inhibits the growth of EGFR-positive cancer cell lines with or without erbB2 overexpression. Int J Cancer 2001; 94:774-82.

19. Leek RD, Hunt NC, Landers RJ, Lewis CE, Royds JA Harris AL. Macrophage infiltration is associated with VEGF and EGFR expression in breast cancer. J Pathol 2000;190:430-6.

20. Grimshaw MJ, Hagemann T, Ayhan A, Gillett CE Binder C, Balkwill FR. A role for endothelin-2 and its receptors in breast tumor cell invasion. Cancer Res 2004;64:2461-8.

21. Sameni M, Dosescu J, Moin K, Sloane BF. Functional imaging of proteolysis: stromal and inflammatory cell increase tumor proteolysis. Mol Imaging 2003;2:159-75.

22. Salvesen HB, Akslen LA Significance of tumourassociated macrophages, vascular endothelial growth factor and thrombospondin-1 expression for tumour angiogenesis and prognosis in endometrial carcinomas. Int J Cancer 1999;84:538-43.

23. Scott BB, Weisbrot LM, Greenwood JD, Bogoch ER, Paige CJ, Keystone EC. Rheumatoid arthritis synovia fibroblast and U937 macrophage/monocyte cell line interaction in cartilage degradation. Arthritis Rheum 1997;40:490-8.

24. Aharinejad S, Paulus $P$, Sioud $M$, et al. Colonystimulating factor-1 blockade by antisense oligonucleotides and small interfering RNAs suppresses growth of human mammary tumor xenografts in mice. Cancer Res 2004;64:5378-84. 